\title{
Bounds on the coverage probability of heterogeneous cellular networks
}

\author{
Morteza Banagar, Behrouz Maham, Vahid Shah-Mansouri
}

- School of Engineering

\begin{abstract}
In a heterogeneous cellular network $(\mathrm{HCN})$, multiple tiers are employed to improve the network coverage probability. For this purpose, different properties of the coverage probability of an HCN should be studied carefully. This paper is classified into two main parts. First, a tight upper bound for the coverage probability of an HCN is derived. It is shown that this upper bound can be used as a good approximation for computing the network coverage probability. At the first glance, one may suppose that adding a new tier to an HCN improves the total coverage probability. In the second part, we study and formulate this problem and investigate the circumstances under which the coverage probability is improved by adding a new tier. Simulation results confirm the validity of our work and show that adding more base stations in the form of a new tier in populated areas can significantly increase the coverage probability, while in less dense areas adding a new tier is not much efficient in terms of improving the coverage probability.
\end{abstract}

Original language

Title of host

publication

Publisher

Pages

Number of pages

ISBN (Electronic)

State

Event

Other

Other

Country
English

2016 IEEE International Conference on Communications

Workshops, ICC 2016

Institute of Electrical and Electronics Engineers Inc.

755-759

5

9781509004485

Published - Jul 52016

2016 IEEE International Conference on Communications

Workshops, ICC 2016 - Kuala Lumpur, Malaysia

2016 IEEE International Conference on Communications

Workshops, ICC 2016

Malaysia 
City Kuala Lumpur

Period $5 / 23 / 16 \rightarrow 5 / 28 / 16$

Banagar, M., Maham, B., \& Shah-Mansouri, V. (2016). Bounds on the coverage probability of heterogeneous cellular networks. In 2016 IEEE International Conference on Communications Workshops, ICC 2016. (pp. 755-759). [7503878] Institute of Electrical and Electronics Engineers Inc.. DOI: $\underline{10.1109 / I C C W .2016 .7503878}$ 